\title{
A Correlative Study Between Size of Tympanic Membrane Perforation, Pure Tone Audiometry and Intraoperative Findings in Tympanoplasty
}

\author{
Santosh U P, ${ }^{1}$ Sridurga $J,{ }^{1}$ Aravind D $R^{1}$
}

\begin{abstract}
Introduction
Chronic otitis media (COM) is the most common and prevalent disease of the middle ear. COM has been defined as a longstanding inflammatory condition of middle ear and mastoid, associated with perforation of the tympanic membrane. Tympanoplasties are common surgeries performed for chronic otitis media in inactive mucosal type.

Any otological surgery may involve a menacel hazard of hearing loss post operatively. In this study, an attempt was made to correlate, size of tympanic membrane perforation, pure tone audiometry and intra-operative findings in tympanoplasties, results were analysed and conclusion drawn.

Materials and Methods

Forty patients attending ENT OPD with chronic otitis media (COM), inactive mucosal type, with conductive hearing loss undergoing tympanoplasties who were willing to participate in the study were selected.

Ear was examined pre-operatively to assess the size of perforation and then, pure tone audiometry (PTA) was done to assess the type of hearing loss and its severity. During tympanoplasty, middle ear was inspected for ossicular status and any other pathology was noted. Later, the size of tympanic membrane perforation, pure tone audiometry and intra operative findings were correlated with each other and analysed.

$\underline{\text { Result }}$

In small and medium sized perforation, PTA and intraoperative findings correlated with each other. Whereas, in large and subtotal perforation, there was no correlation.

Conclusion

In small and medium sized perforation, middle ear inspection may not be necessary. Whereas, in large and subtotal perforation it is necessary.

$\underline{\text { Keywords }}$

Deafness; Infant, Newborn; Hearing Tests; Evoked Potentials, Auditory, Brain Stem; Audiometry, Evoked Response; Otoacoustic Emissions, Spontaneous
\end{abstract}

$\underline{\text { ABSTRACT }}$

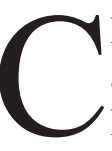
hronic otitis media (COM) is the most common and prevalent disease of the middle ear. ${ }^{1} \mathrm{COM}$ has been defined as a longstanding inflammatory condition of middle ear and mastoid, associated with perforation of the tympanic membrane. ${ }^{2} \mathrm{COM}$ is most commonly a result of acute otitis media. COM with perforation is often accompanied by varying degree of conductive hearing loss. ${ }^{3}$

The surface area of an intact and normally vibrating tympanic membrane plays an important role in transmitting sound energy to middle ear. Perforation of the tympanic membrane is a very common cause of conductive hearing loss as there is loss in the vibrating area of tympanic membrane.

Tympanoplasty is a common surgery, performed for chronic otitis media in inactive mucosal type. The procedure includes inspection of middle ear cleft with

1 - Rajiv Gandhi University of Health Sciences

Corresponding author:

Dr Santosh U P

email: drsantoshup@gmail.com 
Table I: Age distribution

\begin{tabular}{|c|c|c|}
\hline $\begin{array}{c}\text { SOCIO } \\
\text { DEMOGRAPHIC } \\
\text { FACTORS (AGE } \\
\text { IN YEARS) }\end{array}$ & FREQUENCY & PERCENT \\
\hline 20 \& below & 6 & 15 \\
\hline $21-30$ & 19 & 47.5 \\
\hline $31-40$ & 13 & 32.5 \\
\hline $41 \&$ above & 2 & 5 \\
\hline
\end{tabular}

closure of tympanic membrane perforation with a graft (most commonly used graft is autologous temporalis fascia).

In otological surgeries, manipulation of intact ossicular chain may be hazardous; the hearing loss in tympanoplasties is not rare and there are very few publications in this regard. ${ }^{4}$ The post-operative hearing loss may occur due to various reasons. One of the reasons attributed is the manipulation of ossicular chain occurring during the routine inspection of the ossicular chain.

In this study, an attempt was made to correlate, size of tympanic membrane perforation, pure tone audiometry and intra operative findings in tympanoplasties, results were analysed and conclusion drawn.

Chronic otitis media, inactive, mucosal type, with Conductive hearing loss with $38 \mathrm{~dB}$ limit, ${ }^{5}$ Size of tympanic membrane perforation (Small, Medium, Large, Subtotal), Type 1 to Type 3 Tympanoplasties were included in our study.(Fig. 1) Patients not fitting in the inclusion criteria, were excluded from the study.

\section{Materials and Methods}

This prospective study was carried after obtaining institutional ethical committee clearance on a total of 40 patients attending ENT OPD with COM, inactive mucosal type, with conductive hearing loss undergoing tympanoplasties, who were willing to participate in the study were selected, informed and written consent was taken from all patients. The Study period was between August 2016 to June 2017.

Pre-operatively, ear examination was done to visualise size of perforation and then patients were
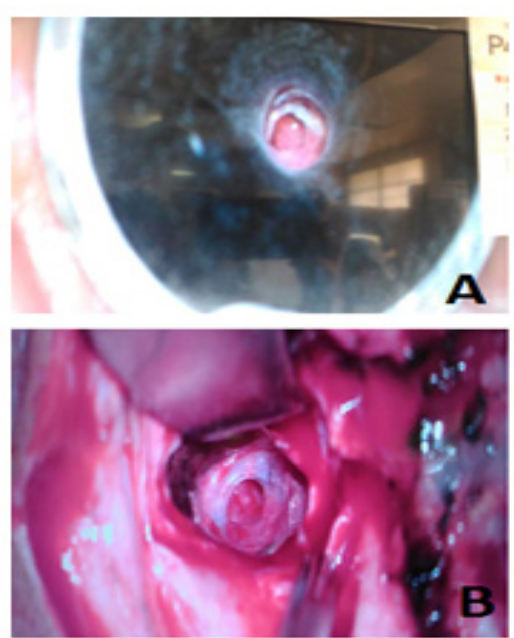
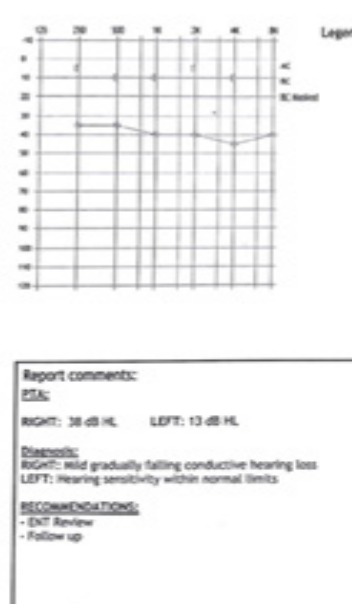
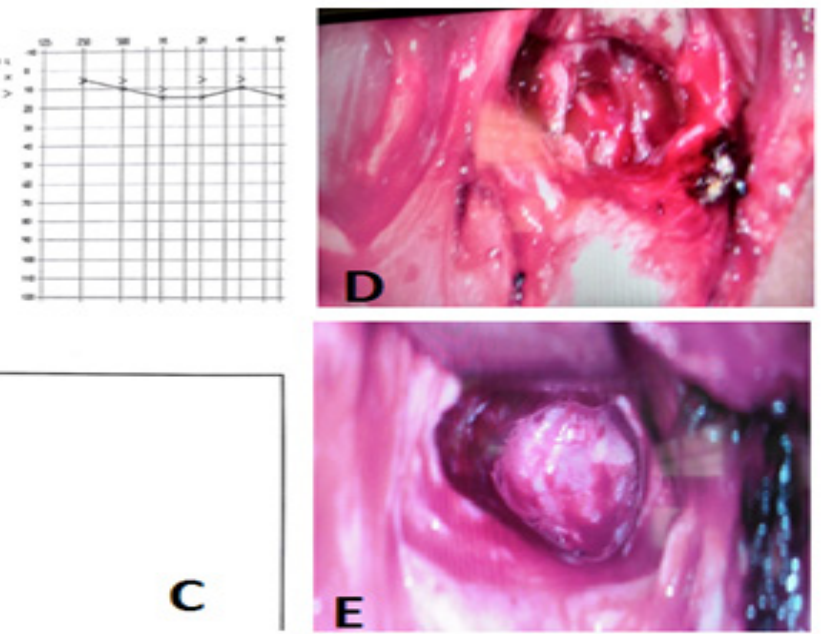

Fig. 1. (A) Tympanic membrane perforation (B) Intra operative picture of tympanic membrane perforation (C) PTA showing mild conductive hearing loss (D) Intra operative picture (E) After placement of temporalis fascia graft. 
Table II: Statistical analysis

\begin{tabular}{|c|c|c|c|c|}
\hline \multirow{2}{*}{$\begin{array}{c}\text { SIZE OF TYMPANIC } \\
\text { MEMBRANE } \\
\text { PERFORATION }\end{array}$} & \multirow{2}{*}{ PTA } & \multicolumn{2}{|c|}{$\begin{array}{l}\text { INTRAOPERATIVE } \\
\text { FINDINGS }\end{array}$} & \multirow{2}{*}{$\begin{array}{c}\text { FISHER'S } \\
\text { EXACT T TEST }\end{array}$} \\
\hline & & $\begin{array}{c}\text { Correlated } \\
(\mathbf{N}=\mathbf{3 3})\end{array}$ & $\begin{array}{l}\text { Not correlated } \\
(\mathbf{N}=7)\end{array}$ & \\
\hline \multirow{2}{*}{ Small \& Medium } & $\begin{array}{l}\text { Correlated } 38 \& \text { Below } \\
\qquad(\mathrm{N}=23)\end{array}$ & 22 & 1 & \multirow{2}{*}{$\begin{array}{c}\text { P }<\mathbf{0 . 0 2 ,}, \\
\text { Significant } \\
\text { CORRELATED }\end{array}$} \\
\hline & $\begin{array}{l}\text { Not correlated Above } 38 \\
\qquad(N=7)\end{array}$ & 4 & 3 & \\
\hline \multirow{2}{*}{ Large \& Subtotal } & $\begin{array}{l}\text { Not Correlated } 38 \& \text { Below } \\
(\mathbf{N}=4)\end{array}$ & 3 & 1 & \multirow{2}{*}{$\begin{array}{c}\text { Not Significant } \\
\text { NOT } \\
\text { CORRELATED }\end{array}$} \\
\hline & Correlated Above $38(\mathrm{~N}=6)$ & 4 & 2 & \\
\hline
\end{tabular}

segregated according to the size of perforation, into four categories as:(a) Small (area involving $<1$ quadrant) (b) Medium (area involving $>1$ and $<2$ quadrants) (c) Large (area involving $>2$ and $<3$ ) (d) subtotal(involving all 4 quadrants). ${ }^{6}$

Pure tone audiometry was done to assess the severity and type of hearing loss.

These tympanoplasties were operated by a single surgeon to maintain uniformity. Under general anaesthesia, through post aural approach, temporalis fascia graft was harvested, external auditory canal entered and tympanic membrane visualised,freshening of margin was done. Tympanomeatal flap elevated, middle ear inspected for ossicles and any other pathology was noted (e.g. fibrosis/ adhesions/ sclerosis/ any other). Temporalis fascia graft was placed by underlay technique, secured with gel foam, wound closed in layers, mastoid dressing was done and regular post-operative care was given.

Later, the size of tympanic membrane perforation, pure tone audiometry and intra operative findings were correlated with each other and analysed.

\section{Result}

In our study, patients were in the age group of 16 to 45 years. Maximum number of patients were between 21 to 30 years of age.(Table I) Fifteen patients (37.5\%) in this series were male twenty-five $(62.5 \%)$ female.

In our study, it was found that, in 23 patients with small and medium sized tympanic membrane perforation, with a PTA of $38 \mathrm{~dB}(\mathrm{AB}$ gap) and below,

Table III: Showing correlation between size of tympanic membrane perforation and intra operative findings Nc*- Ossicular Necrosis, G*- Glue in middle Ear, F*- Ossicular Fixity

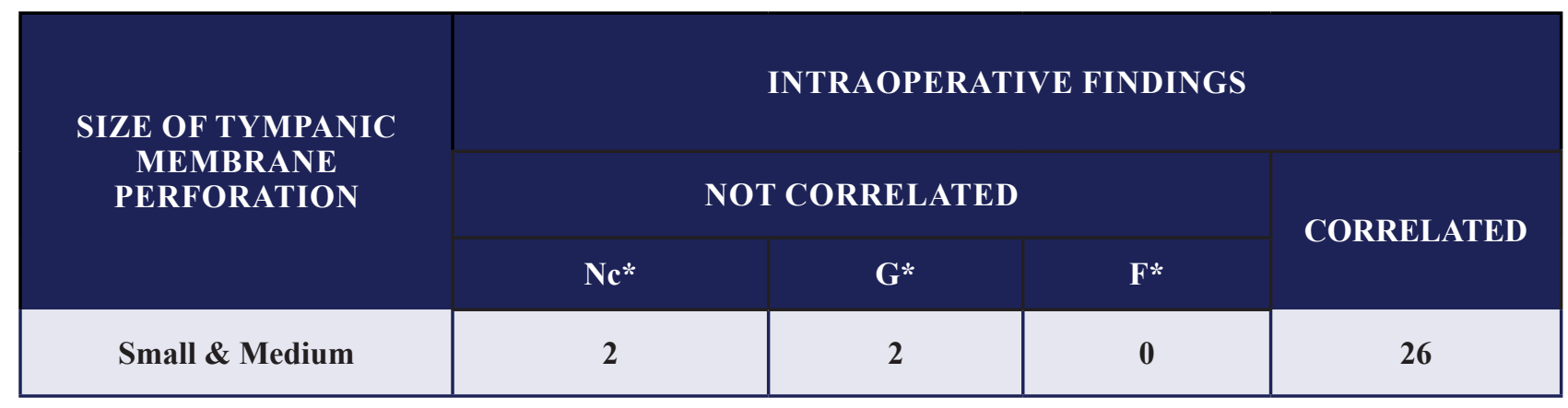


Table IV: Correlation between size of tympanic membrane perforation and intra-operative findings Nc*- Ossicular Necrosis, G*- Glue in middle Ear, F*- Ossicular Fixity

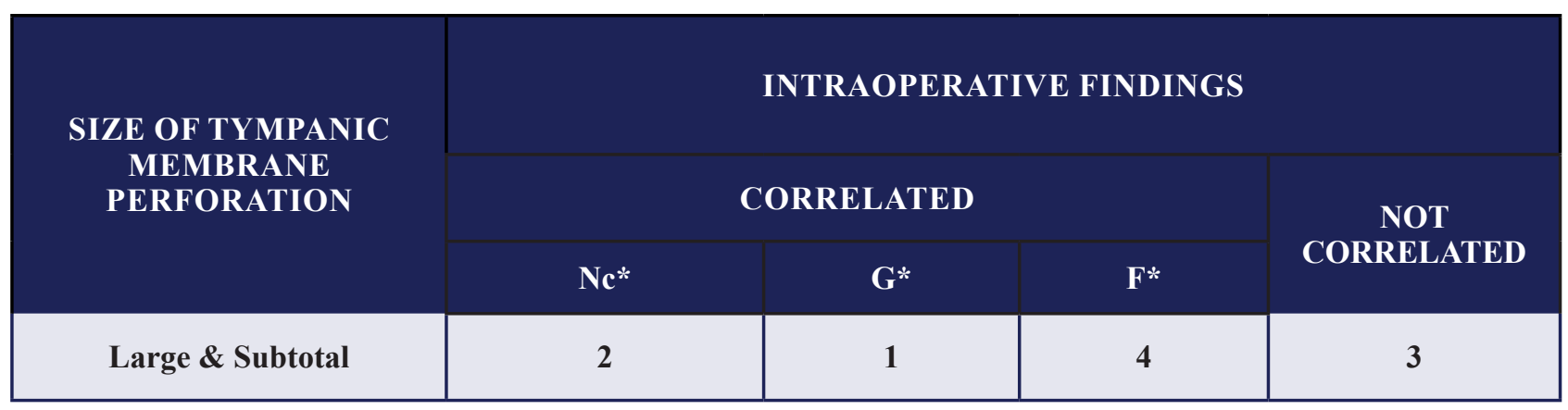

size of perforation correlated with PTA findings. Out of these 23 patients ( 100 per cent), in 22 patients (95.6 per cent), size of tympanic membrane perforation, PTA findings and intra operative findings correlated with each other. In 1 patient (4.4 per cent) the size of tympanic membrane perforation and PTA findings correlated, but intra operative finding did not correlate and showed necrosis of the Incus.(Table II and III)

In 7 patients, with small and medium sized tympanic membrane perforation, with a PTA of above $38 \mathrm{~dB}$, size of tympanic membrane perforation did not correlate with PTA findings. Out of these 7 patients (100 per cent), in 4 patients (57.1 per cent), size of tympanic membrane perforation, did not correlate with PTA findings, but correlated with intra operative findings. In 3 patients ( 42.9 per cent) the size of tympanic membrane perforation, PTA findings and intra operative finding did not correlate, among these 3 patients One patient had necrosis of the incus and two patients had glue in the middle ear.(Table III)

Applying Fisher's exact $\mathrm{t}$ test, the $\mathrm{P}$ value was $<0.02$ (in small and medium size perforation, with PTA and intra operative findings), which is significant.(Table II)

In 4 patients, with large and subtotal sized tympanic membrane perforation, with a PTA of $38 \mathrm{~dB}$ and below, size of tympanic membrane perforation did not correlate with PTA findings. Out of these 4 patients (100 per cent), in 3 patients ( 75 per cent), size of tympanic membrane perforation, did not correlate with PTA findings, but correlated with intra operative findings as expected, among these 3 One had necrosis of the handle of malles and 2 had ossicular chain fixity[one had fixed incudomalleolar joint and other had fixed incudomalleolar and incudostapedial joint].(Table IV)In 1 patient ( 25 per cent) the size of tympanic membrane perforation, PTA findings and intra operative finding did not correlate.

In 6 patients with large and subtotal sized tympanic membrane perforation, with a PTA of above $38 \mathrm{~dB}$, size of tympanic membrane perforation correlated with PTA findings. Out of these 6 patients (100 per cent), in 4 patients (66.7per cent), size of tympanic membrane perforation, PTA findings and intra operative findings correlated with each other as expected, among these 4 patients one has necrosis of incus one had glue in middle ear and two had ossicular fixity [ both had incudostapedial joint fixed]. (Table IV) In 2 patients (33.3 per cent) the size of tympanic membrane perforation and PTA findings correlated, but intra operative finding did not correlate.

Applying Fisher's exact $t$ test, the P value was not significant (in large and subtotal size perforation, with PTA and intra operative findings).

Using the software IBM SPSS version 22 for windows, the above statistical analysis was done.

\section{Discussion}

COM (Tympanic membrane perforation)is a very common disorder caused either by infection or trauma, resulting in decreased hearing. So, a meticulous examination, diagnosis and treatment of tympanic membrane perforation are necessary for the assessment 
and restoration of hearing loss.

According to the study done by Nepal et al, ${ }^{7}$ hearing loss was greater in larger perforations as compared to small perforations. But in our study, we found that, in small and medium sized perforations, size of tympanic membrane perforation correlates with PTA results, whereas in large and subtotal perforation it was not correlating.

Similarly, in a study done by Singh et al, ${ }^{8}$ concluded that the hearing loss is directly proportional to the size of the perforation. But in our study we found the above mentioned observation.

In a study done by Ribeiro et $\mathrm{al}^{,}{ }^{9}$ there was no significant relationship between the size of tympanic perforations and hearing loss.

This is a unique study, in which three variables are correlated (size of tympanic membrane peroration, PTA and intra operative findings).

Routinely during surgery, while inspecting the middle ear, the chances of manipulation of the ossicular chain is there, causing deterioration of post-operative hearing. A detailed correlative study is necessary in this regard, because even in a straight forward case, any manipulation during the inspection of an intact ossicular chain might be hazardous. ${ }^{3}$ However, this study has to be conducted with a larger sample size.

\section{Conclusion}

In small and medium sized perforation, PTA and intraoperative findings correlated with each other. Whereas, in large and subtotal perforation, there was no correlation.

In small and medium sized perforation, middle ear inspection may not be necessary. Whereas, in large and subtotal perforation it is necessary.

\section{References}

1. Kumar N, Chilke D, Puttewar M.P. Clinical profile of tubotympanic CSOM and its management with special reference to site and size of tympanic membrane perforation, Eustachian tube function and three flap tympanoplasty. Indian J Otolaryngol Head Neck Surg. 2012; 64(1): 5-12

2. Lerut B, Pfammatter A, Moons J, Linder T. Functional correlation of tympanic membrane perforation size. Otol Neurotol. 2012; 33(3):379-86

3. Kaur K, Chadha S, Kumar D and Preethi. Evaluation of hearing loss in tympanic membrane perforation. Indian J Otolaryngol Head Neck Surg. 2011; 63(3): 208-13

4. Bewitch J, Prinsley P. Hearing loss following myringoplasty implications of informed consent. The Journal of Laryngol \& Otol. 2015; 129:342-7

5. Dhingra PL. Dhingra S. Disease of ear, nose and throat. 5th edn. New Delhi. Elsevier.2010. Chapter 5. pp 34

6. Nepal A, Bhandary S, Mishra SC, Singh I, Kumar P. The morphology of central tympanic membrane perforations. Nepal Med Coll J. 2008;9:239-44

7. Nepal A, Bhandary S, Mishra SC, Singh I, Kumar P. The morphology of central tympanic membrane perforations. Nepal Med Coll J. 2008;9:239-44

8. Singh R, Srivastava A, Mohan C. Comparative evaluation of hearing in different tympanic membraneperforations. Indian $\mathrm{J}$ Otol. 2016;22:258-61

9. Ribeiro FA, Gaudino VR, Pinheiro CD, Marc, al GJ, Mitre EI. Objective comparison between perforation andhearing loss. Braz J Otorhinolaryngol. 2014;80:386-9. 